\title{
Demonstration of the Source of Motor Program Signal: Study on the Correlation between Muscle Strength and sEMG Signal in Normal Children and Adults
}

\author{
Ming Qi¹, Xiujuan Xie ${ }^{1}$, Haiying Pang11, Yujie Sun ${ }^{1}$, Chengqian Fang ${ }^{1}$, Wenru Zhao ${ }^{1,2}$ \\ ${ }^{1}$ Reasearch Room of Rehabilitation of Neurological Training, Beijing Daxing District Hospital of Chinese and \\ Western Medicine, Beijing, China; ${ }^{2}$ Institute of Neurological Training Rehabilitation Medicine, Sanya Hongsen \\ Hospital, Harbin Medical University, Sanya, China
}

Correspondence to: Wenru Zhao, zhaowenru7006@sina.com

Keywords: Rehabilitation, Motor Program Signal, Source, Mechanism Demonstration

Received: April 27, $2021 \quad$ Accepted: June 5, $2021 \quad$ Published: June 8, 2021

Copyright $\odot 2021$ by author(s) and Scientific Research Publishing Inc.

This work is licensed under the Creative Commons Attribution International License (CC BY 4.0).

http://creativecommons.org/licenses/by/4.0/

\section{(c) (i) Open Access}

\section{ABSTRACT}

To investigate the relationship between muscle strength and sEMG of biceps brachii during elbow flexion by measuring the maximum muscle strength and sEMG value of normal children and adults, and to analyze their sources, so as to lay a theoretical foundation for the method of motor program reconstruction to restore the function after brain injury, 30 healthy children aged $9-10$ years and 30 adults aged $20-30$ years were randomly selected. The muscle strength and sEMG of biceps brachii during elbow flexion were detected and recorded, and the data were statistically analyzed. The muscle strength of children was significantly lower than that of adults $(P<0.001)$, and the sEMG value of biceps brachii was significantly lower than that of adults $(P<0.001)$, but the sEMG value per kilogram force of children was significantly higher than that of adults $(P<0.01)$. The results show that there was a very significant difference in pull (efficiency) between adults and children when there was no significant difference in SEMG signal intensity. This is because although children's central nervous system has matured, the muscle tissue has not been well trained, resulting in insufficient muscle strength. The muscle strength of adults is significantly higher than that of children, because they have been exercising for a long time after the development of the central nervous system. It is proved that sEMG signal is not produced by muscle contraction itself, but comes from the motor program signal of central nervous system which drives muscle contraction, and it is produced before muscle contraction.

\section{INTRODUCTION}

Some studies have shown that [1] motor program reconstruction technology has better recovery of 
motor function lost after brain injury, and animal experimental studies have confirmed that [2] the electrical signal received on the skin surface of muscle contraction is the motor program signal driving muscle contraction, which mainly comes from the motor center of cerebral cortex rather than the muscle itself.

But do motion programs really exist? If so, where does the signal come from? Whether the electrical signal received on the skin surface of muscle contraction is generated by muscle contraction itself still needs to be further demonstrated, so as to lay a theoretical foundation for the research and clinical application of motor program reconstruction theory and method.

In the past, it was generally believed that the electrical signal received during muscle contraction was produced by muscle contraction itself. If so, the signal should increase in proportion with the increase of muscle strength. Although the development of children is not complete and the muscle strength is weak, the sEMG signal intensity should be increased in a positive proportion to the enhancement of muscle strength. If it does not conform to this rule, for example, when the sEMG signal is relatively constant and the muscle strength changes obviously, it means that the sEMG signal is not produced by the muscle contraction itself, but from the driving signal of the central nervous system which controls the muscle contraction. Just like the working principle of the spark plug in the cylinder and the power generated by fuel gas combustion, the acceleration of the vehicle is realized by increasing the fuel supply, rather than by increasing the amount of spark injected by the spark plug. Human tissues are different from mechanical devices. When the amount of muscle tissue controlled by the brain increases, the number of brain cells that are activated to participate in the exercise will also increase, and the intensity of the control signal will be different in a certain range.

On the basis of previous studies, this study observed the correlation between the strength of biceps brachii muscle and the intensity of sEMG signal in children and adults with different intensities of active elbow flexion, in order to further clarify the source of sEMG signals.

\section{METHODS}

Participants: 30 healthy primary school students of Grade 5 - 6 were randomly selected, including 13 boys and 17 girls, with an average age of 9.5 years old; 30 healthy adults of the hospital staff were randomly selected, including 16 males and 14 females, with an average age of 24.6 years.

Before the test, the subjects were informed of the whole trial process in detail, and informed consent was signed. Explain the precautions and operation methods in detail, and carry out experimental practical operation and correct incorrect methods, so that each subject could fully grasp the action essentials. During the test, the subjects were required to fully relax and avoid compensatory force from other parts of the body during the test.

\section{Procedures}

Skin preparation: disinfect and degrease the skin surface with medical alcohol cotton ball to reduce the contact resistance between electrode and skin.

Equipment: Neuroeductor III electromyographic biofeedback therapeutic apparatus and $3 \mathrm{M} \mathrm{Ag}$ $\mathrm{AgCl}$ surface electrode were used. Neuroeducator III is used to receive and display sEMG signals received during elbow flexion in real time. The signal is based on the square of the root mean value of the potential with noise level less than $0.2 \mu \mathrm{V}$, and the sEMG signal with a bandwidth of $10-1000 \mathrm{~Hz}$ is analyzed, and combined with more than $0.1 \mathrm{~s}$, it is standardized into $\mu \mathrm{Vs}$, and the current data of every $1 / 10 \mathrm{~s}$ is displayed on the color display in the form of continuous curve. The scanning time of each screen is $20 \mathrm{~s}$, and the data is stored in the database for analysis.

The subjects sit on the chair, hold their chest and head up, put the palms of their right hands up on their thighs, breathe well and relax fully. The reference electrode was pasted with the most prominent part of biceps brachii as the midpoint, and the receiving electrode was pasted $4 \mathrm{~cm}$ each away from the distal end of the electrode, and connected to the first lead of neuroeductor III. The baseline of sEMG signal was adjusted to zero position, and then the attention was focused on the biceps brachii, and the following tests were carried out respectively. 
Elbow flexion without resistance: stretch the elbow to 90 degrees with maximum force and maintain it for 5 seconds. Meanwhile, the sEMG signal intensity was detected, and the average value was recorded for 3 consecutive times.

Maximum elbow flexion test: AFG50 push-pull electronic tensiometer was used. The subjects held the handle of the extensometer, and the other end was vertically fixed under the feet of the subjects. The subjects did elbow flexion with maximum pulling force to 90 degree position for 5 seconds and repeated 3 times. At the same time, the pulling force value and sEMG signal value are recorded, and the average value is input into the database for analysis.

\section{Statistical analysis}

SPSS17.0 statistical software was used to analyze the experimental data. The two groups of data were normally distributed. The t-test of two independent samples showed that one group of the two groups did not belong to the normal distribution, and the test of non-parametric rank sum was adopted.

\section{RESULTS}

The maximum Muscle Strength (MS) of adults was $21.50 \mathrm{~kg}$, and the sEMG intensity was $542.00 \pm$ $200.47 \mu \mathrm{V}$, while that of children was $7.04 \pm 4.84 \mathrm{~kg}$ and the sEMG intensity was $262.67 \pm 111.94 \mu \mathrm{V}$. The muscle strength of adults was 3.05 times of that of children, and the sEMG intensity of adults was 2.06 times of that of children. The sEMG signal intensity required for $1 \mathrm{~kg}$ force produced by muscle contraction in adults was $28.33 \mu \mathrm{V}$, while that in children was $47.46 \mu \mathrm{V}$, almost twice that of adults $(\mathrm{P}<0.01)$, See Table 1 and Figure 1.

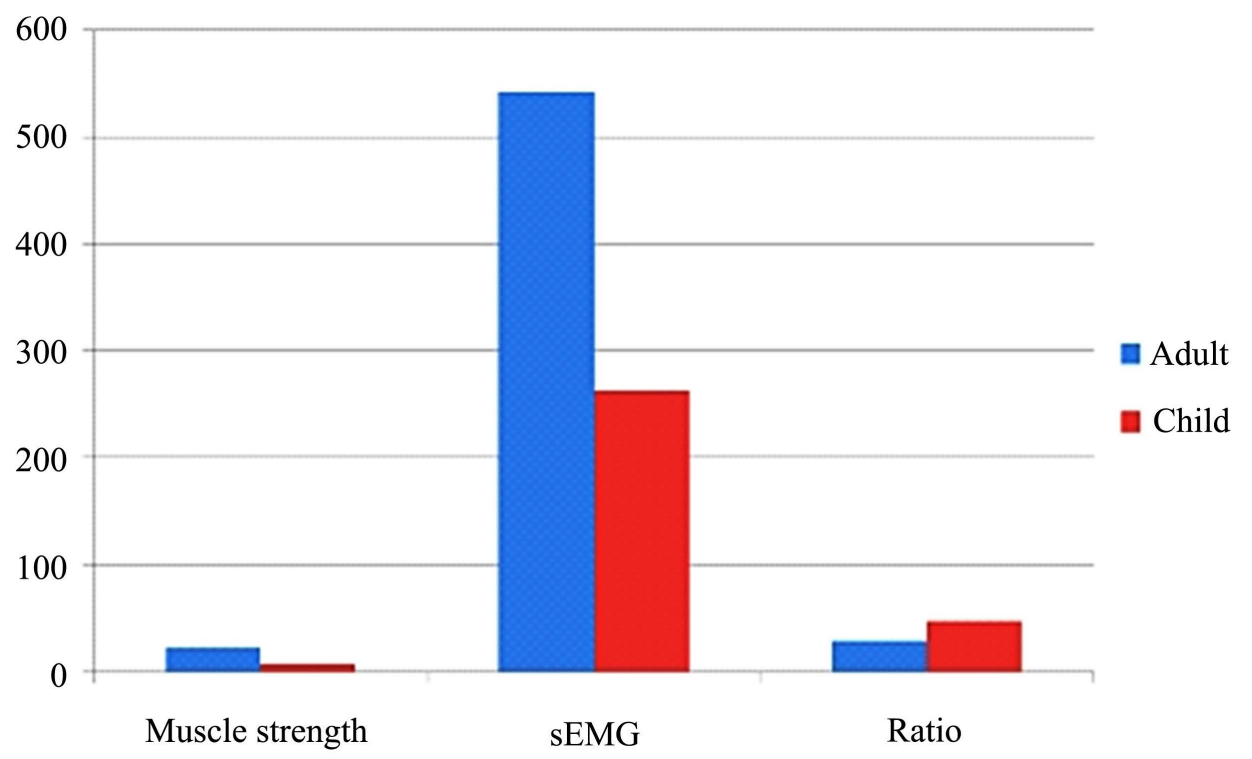

Figure 1. Comparison of muscle strength, EMG signal and their ratio between adults and children.

Table 1. Statistical analysis of MS and sEMG results of biceps brachii in children and adults during elbow flexion.

\begin{tabular}{cccc}
\hline Item & Children & Adults & P \\
\hline MS $(\mathrm{Kg})$ & $7.04 \pm 4.84$ & $21.50 \pm 10.04$ & $<0.001$ \\
sEMG $(\mu \mathrm{V})$ & $262.67 \pm 111.94$ & $542.00 \pm 200.47$ & $<0.001$ \\
sEMG/MS $(\mu \mathrm{V} / \mathrm{Kg})$ & $47.46 \pm 23.03$ & $28.33 \pm 12.47$ & $<0.001$ \\
\hline
\end{tabular}


Muscle strength only increased with the increase of sEMG in a certain range, but not in a positive proportion. When sEMG reached a certain range (about $640 \mu \mathrm{V})$, the sEMG did not increase significantly no matter how strong the muscle strength was; children's sEMG is stronger than muscle strength, while adult's muscle strength was stronger than sEMG, which indicated that the development of CNS producing sEMG was earlier than that of muscle producing muscle strength. The development of brain cells that control movement was relatively mature at about 3 years old after birth, and the enhancement of muscle strength needed to gradually make muscle fiber coarsen and increase muscle strength during long day-to-day work, training and self-exercise.

When driving muscle contraction between adults and children, the difference between driving signals (sEMG) was small, but the difference between muscle strength was huge, which could also be explained by the phenomenon of high and low power of electrical appliances. At a rated voltage of $220 \mathrm{~V}$, a 100 watt bulb was much brighter than a 10 watt bulb. Although the voltage of the two were the same, the effect difference caused by the different power of the effector was very obvious. The voltage intensity of the motion program signal from the human central nervous system to the muscles may have the function of automatic regulation. It could determine the voltage intensity of driving signal according to the actual demand of motion intensity. Under the same electric signal intensity, there would be different muscle strength differences. The stronger the muscle strength, the greater the energy consumption, otherwise, the less energy consumption.

\section{DISCUSSION}

At present, it is generally believed that the electrical signal received on the skin surface of muscle contraction is produced by muscle contraction itself. If so, training to increase the intensity of the signal is to increase muscle strength, not brain function. However, the limb motor dysfunction caused by brain injury is only the external manifestation after brain injury, and there is no problem with the limbs themselves. Just as the generator of a power plant is broken and cannot generate electricity, which leads to the light bulb not lighting, the basic reason is that the generator is damaged. Therefore, the motor function lost after brain injury must be restored on the basis of recovery of brain function. The purpose of this experiment is to further understand the source of sEMG, to understand whether the motor program exists, and whether the brain function can be restored by motor program reconstruction technology, and to lay a theoretical and methodological foundation for the clinical application.

Xin Li et al. [3] stimulated the soles of healthy adult New Zealand rabbits with acupuncture needles to cause the autonomic contraction of gastrocnemius muscles of rabbits. Neuroeductor III produced by American Therapeutic Alliances company was used to detect and record the ssEMG. The results showed that under normal conditions, strong signals could be detected when the gastrocnemius muscle was actively contracted by stimulating the sole of the foot; Although the gastrocnemius muscle contraction was induced by the stimulation of the sole of the foot after the spinal cord was severed from the lumbar 1 level of the rabbit, it belonged to transient reflex (similar to knee tendon reflex), and the weak transient reflex signal was detected; After the sciatic nerve was cut off, repeated stimulation of the sole of the foot did not cause gastrocnemius muscle contraction, and no signal was detected. It is confirmed that the electrical signals received on the skin surface of muscle contraction are produced in the brain and spinal cord, mainly from the motor center of cerebral cortex. It indicates that the generation of limb voluntary movement is a nerve impulse signal from the motor center of the cerebral cortex, which is transmitted to the peripheral nerve through the motor neurons of the anterior horn of the spinal cord, and then causes muscle contraction [4]. When the motor center of cerebral cortex is damaged or the conduction pathway is interrupted, even if the muscle structure is intact, the contraction process will not occur. It is proved that the relationship between brain and limb movement is close, and many studies have also confirmed the synchronous correlation between the two [5-9]. Wenru Zhao et al. [10] found that every movement of the human body is driven and controlled by the signals from CNS cells to the muscle, which is generated before muscle contraction, rather than muscle contraction itself. The signal is sent in different proportion 
and order, which is called motion program. The movement program controls the regular contraction and relaxation of muscles involved in joint activities and limb movements, which makes the human body move in a coordinated and orderly manner, and has the actual function of completing a certain action.

The results showed that, in both children and adults, with the increase of muscle strength, the sEMG signal value of biceps brachii increased gradually, but it only increased in a certain range in a positive proportion, and then showed that with the increase of muscle strength, the amplitude of sEMG signal value was becoming smaller and smaller. Compared with adults, the ratio of sEMG signal to muscle strength in children is significantly higher than that in adults under the condition of individual maximum tension. This may be due to the fact that children's brain develops earlier and muscles develop later due to lack of exercise, which further proves that the electrical signals received from the skin surface of muscles during muscle contraction are from the CNS system but not produced by the muscle itself.

Although the enhancement of muscle strength is directly related to the improvement of brain function, in many cases, the enhancement of muscle strength is not the result of the improvement of brain function. Because muscle strength belongs to the effect produced by effector, and the effect can be changed obviously. Such as long-term bedridden people, because of the muscle cannot be used and cause muscle atrophy, resulting in muscle strength decline. A regular physical exercise of the people, will make muscle strength has been significantly improved. For example, a weight lifter can lift 100 kilograms after one year's training. However, if he does not exercise for two years, he may not even be able to lift $50 \mathrm{~kg}$, but his brain function has not significantly decreased. The establishment of function in the brain is completely different, because it is a process of learning methods, once learned, unless the brain tissue is damaged, it is not easy to lose. For example, a person who has learned how to swim and ride a bicycle can still ride and swim after five years without riding or swimming. However, when he starts swimming again or riding a bicycle, his proficiency is slightly poor. This is because the brain has learned how to ride a bicycle and swim, which is the establishment of a methodology. This methodology is actually the process in which the brain controls the limbs to complete a certain movement, that is, the motor program. The signal of the motor program is transmitted to the muscles involved in the movement step by step to drive the active muscle to contract and the antagonistic muscle to relax properly at the same time, so as to complete the limb movement formed by flexible joint activities or multi joint activities. Moreover, the motor program signal can be received and displayed on the fluorescent screen with the help of biological signal receiving equipment, which lays a theoretical and methodological foundation for the training of abnormal motor program correction and normal motor program reconstruction.

In addition, we have also measured the sEMG of various parts of the human body, and found that only two parts can be received. One is the skin surface located of the muscle, which comes from the motor center of cerebral cortex, which is a movement program signal to drive skeletal muscle contraction. The other is located on the surface of the chest wall of the heart. The signal comes from the sinoatrial node above the right atrium, which is an involuntary motion program signal to drive myocardial contraction. In addition, the sEMG signal was not received in other parts of the body. For example, the electrical signal of peristaltic contraction of gastrointestinal smooth muscle was not received in the abdominal wall, and the electrical signal of arterial pulsation was not received in the neck and groin. Special equipment may be needed to detect it, but it needs further research.

Application of traditional Chinese Medicine Daoyin-feedback rehabilitation technology [11] to develop brain potential in order to using the spare brain cells. The neurological training instrument is used to receive the guided motor program signal in real time and display it in the form of digital curve [12]. On the basis of correcting abnormal motor program, the training of reconstructing normal motor program is implemented, and satisfactory clinical effect has been achieved [13].

\section{CONCLUSION}

It is proved that sEMG signal is not produced by muscle contraction itself, but comes from the motor program signal of central nervous system which drives muscle contraction, and it is produced before mus- 
cle contraction.

\section{ACKNOWLEDGEMENTS}

Ms. Lijuan Fei, Secretary General of neurorehabilitation branch of Chinese medicine information society, organized students to participate in the experiment, which made the experiment successfully completed.

\section{AUTHORS CONTRIBUTION}

1) Corresponding author Wenru Zhao has conceived this research, by referring to file and looking up new retrieval, raised the deep mechanism and wrote this thesis. 2) Ming Qi. Participate in treatment, observe patients, collect data, make statistical analysis and drafting the paper; 3) Haiying Pang, Yujie Sun, Fang Chengqian participated in treatment and collect data.

\section{CONFLICTS OF INTEREST}

The authors declare no competing interests.

\section{REFERENCES}

1. Li, Z. (2006) Signal Analysis of Electromyogram and Its Application in Sport Scientific Research. Bulletin of Sport Science and Technology, 14, 31-34.

2. Hagg, G.M. (1992) Interpretation of sEMG Spectral Alterations and alteration Indexes at Sustained Contraction. Journal of Applied Physiology, 73, 1211-1217. https://doi.org/10.1152/jappl.1992.73.4.1211

3. Li, X., Zhao, H.-H., Sun, A.-P., Zhang, X.-M., Cao, X. and Zhao, W.-R. (2011) Relations of Surface Electromyogram Signal with Muscle Contraction and Motor Centers in Rabbit Gastrocnemius Muscle. Journal of Clinical Rehabilitative Tissue Engineering Research, 15, 8693-8697.

4. Yao, T., Editor. (2001) Physiology. People's Health Press, Beijing, 50-51.

5. Boonstra, T.W., van Wijk, B.C., Praamstra, P., et al. (2009) Corticomuscular and Bilateral sEMG Coherence Reflect Distinct Aspects of Neural Synchronization. Neuroscience Letters, 463, 17-21. https://doi.org/10.1016/j.neulet.2009.07.043

6. Szucs, D., Soltész, F. and White, S. (2009) Motor Conflict in Stroop Tasks: Direct Evidence from Single-Trial Electro-Myography and Electro-Encephalography. Neuroimage, 47, 1960-1973. https://doi.org/10.1016/j.neuroimage.2009.05.048

7. Mima, T., Matsuoka, T. and Hallett, M. (2001) Information Flow from the Sensorimotor Cortex to Muscle in Humans. Clinical Neurophysiology, 112, 122-126. https://doi.org/10.1016/S1388-2457(00)00515-0

8. Gagné, M., Reilly, K.T., Hétu, S., et al. (2009) Motor Control over the Phantom Limb in Above-Elbow Amputees and Its Relationship with Phantom Limb Pain. Neuroscience, 162, 78-86.

https://doi.org/10.1016/j.neuroscience.2009.04.061

9. Priori, A., Bertolasi, L., Dressler, D., et al. (1993) Transcranial Electric and Magnetic Stimulation of the Leg Area of the Human Motor Cortex: Single Motor Unit and Surface sEMG Responses in the Tibialis Anterior Muscle. Electroencephalography and Clinical Neurophysiology, 89, 131-137 https://doi.org/10.1016/0168-5597(93)90095-7

10. Zhao, W.R., Editor. (2014) Rehabilitation Therapeutics of Neurological Training. People's Medical Publishing House, Beijing.

11. Zhao, W.R., Zhang, P.C. and Qi, M. (2019) Combined Rehabilitation Method and Its Mechanism of Traditional Chinese Medicine Daoyin Technique with Biofeedback Technique. Journal of Biomedical Science and Engi- 
neering, 12, 652-656. https://doi.org/10.4236/jbise.2019.1212042

12. Zhao, W.R., Editor. (2019) Rehabilitation Therapeutics of Neurological Training: Daoyin Technique in Chinese Medicine. Springer-People's Medical Publishing House, Beijing. https://doi.org/10.1007/978-981-13-0812-3

13. Zhao, H.H., Sun, A.P., Zhang, X.M., Wang, Z., Cao, X., Li, X. and Zhao, W.R. (2011) Rehabilitating Effect and Mechanism of Neurological Training of Genurecurvatmn in Hemlplegic Patient. China Medicine, 6, 476-478. 